\title{
NEW ASPECTS IN THE TREATMENT OF HYPERTENSION
}

\author{
Viktor Meretskyi \\ Department of Pharmacology and Clinical Pharmacology of I.Ya. Horbachevsky Ternopil State Medical University
}

Meretskyi V. (2015), New aspects in the treatment of hypertension. Health Problems of Civilization, 3 (9), p. 16-19.

\begin{abstract}
Summary: Hypertension is currently the leading cause of cardiovascular complications (heart attack, stroke) and the resulting deaths of patients. Reduced morbidity and mortality from cardiovascular complications is the main goal of treating patients suffering from high blood pressure (BP). To achieve target BP levels in the arsenal of physicians are five major classes of antihypertensive drugs: angiotensin-converting enzyme inhibitors, angiotensin II receptor blockers, calcium channel blockers, $\beta$-blockers, diuretics. The choice of antihypertensive drug in concrete clinical situation often complicated and determined by complex factors. Among these presence of risk factors; target organ damage; associated clinical conditions, metabolic syndrome, diabetes, comorbidities; possible individual patient response to antihypertensive drugs of different classes in history; the likelihood of drug interactions; socio-economic factors, including the cost of treatment of hypertension. Promising is the use of angiotensin II receptor blockers - drugs with pleiotropic pharmacological properties that have a multicomponent antihypertensive efficacy, good tolerability, diverse organoprotection that are safe, able to enhance remote prognosis for patients with hypertension. Azilsartan medoxomil is a competitive reversible angiotensin II type 1 receptor antagonist. Azilsartan developing hypotensive effect faster, prolonged and pronounced compared to other sartans (valsartan, candesartan and olmesartan). In addition to antihypertensive action azilsartan medoxomil shows a number of additional pleiotropic effects. These include antithrombotic, antiproliferative and antyfibrotic action. It demonstrates the improvement in glucose tolerance and tissue insulin sensitivity, improves endothelial function, reduces the progression of albuminuria, proteinuria.
\end{abstract}

Keywords: hypertension, angiotensin II receptor blockers, azilsartan medoxomil

\section{Introduction}

Hypertension is an epidemic affecting one billion people and is the commonest risk factor for death throughout the world. Increased attention to hypertension based on the results of large-scale epidemiological and clinical studies have repeatedly demonstrated adverse effects of elevated blood pressure (BP) on the risk of cardiovascular events, including death from cardiovascular causes (Dyadyk et al. 2014, Mancia et al. 2013).

Limited comparable data are available on the prevalence of hypertension and the temporal trends of BP values in different European countries. Overall the prevalence of hypertension appears to be around 30-45\% of the general population, with a steep increase with ageing (at least $60 \%$ of people over the age of $60-65$ years have high BP or receiving antihypertensive treatment). Among persons aged 55-65 years, the likelihood of developing hypertension, according to the Framingham study, is more than $90 \%$. The World Health Organization (WHO) considers hypertension as the most important preventable causes of worldwide death. Hypertension is associated with increased cardiovascular mortality and risk of cardiovascular events in all age groups; among the elderly degree of risk has a direct relationship with the level of systolic blood pressure and feedback to the level of diastolic blood pressure. A close relationship between prevalence of hypertension and mortality for stroke has been reported. The incidence and trends of stroke mortality in Europe have been analysed by use of WHO statistics. Western European countries exhibit a downward trend, in contrast to eastern European countries, which show a clear-cut increase in death rates from stroke (Mancia et al. 2013, New therapeutic possibilities of control of hypertension: a modern angiotensin receptor antagonist Edarbi (azilsartan medoxomil) 2014).

Search of therapeutic approaches that can prevent the patient consistent transition from one stage of cardiovascular continuum to the other, is now a priority in cardiology. The most significant positive result in this task can be expected from an effective intervention in the early stages of the mentioned pathological process

Address for correspondence: Viktor Meretskyi, I.Ya. Horbachevsky Ternopil State Medical University, m.Voli, 1, Ternopil, 46001, e-mail: meretskyivm@gmail.com, phone: +38 0992376857

Tables: 0 Figures: 0 References: 21 Full-text PDF www.hpc.edu.pl Copyright (C) Pope John Paul II State School of Higher Education in Biała Podlaska, Sidorska 95/97, 21-500 Biała Podlaska Indexation: Index Copernicus, AGRO, ProQuest, Polish Medical Bibliography, Polish Ministry of Science and Higher Education. This is an open-access article distributed under the terms of the Creative Common Attribution Non-commercial license (http://creativecommons.org/licenses/by-nc/3.0), which permits use, distribution and reproduction in any medium, provided the original works is properly cited, the use is non-commercial and is otherwise in compliance with the license. 
that involves the impact on risk factors, including normalization of blood pressure (Svischenko et al. 2012, Ostroumova et al. 2010). Reduced morbidity and mortality from cardiovascular complications is still the main goal of treating patients suffering from high blood pressure. To achieve target BP levels in the arsenal of physicians are five major classes of antihypertensive drugs: angiotensin-converting enzyme (ACE) inhibitors, angiotensin II receptor blockers (ARBs), calcium channel blockers, $\beta$-blockers, diuretics. The results of a multicenter randomized trials proved that none of them has significant advantages in terms of severity of antihypertensive effect. The choice of antihypertensive drug in concrete clinical situation often complicated and determined by complex factors. Among these presence of risk factors; target organ damage; associated clinical conditions, metabolic syndrome, diabetes, comorbidities; possible individual patient response to antihypertensive drugs of different classes in history; the likelihood of drug interactions; socio-economic factors, including the cost of treatment of hypertension (Mancia et al. 2013, Chazova et al. 2011).

According to modern concepts activation of the renin-angiotensin-aldosterone system (RAAS) plays a critical role in the regulation of blood pressure. Angiotensin II (AT II) as a key effector hormone of the RAAS via vasoconstriction, an increase in left ventricular afterload, retention of sodium and water increases BP and helps to stabilize hypertension. Long-term effects of elevated levels of angiotensin II, especially in tissues, leading to cardiac and vascular remodeling, kidney disease, which also contributes through organ violations increased risk of cardiovascular disease and renal failure (Chazova et al. 2011, Korost et al. 2014).

The first drugs that block the RAAS was nonselective peptide antagonist of AT II receptor, which was called "saralasin". This peptide structurally similar to AT II, acted as a competitive inhibitor of angiotensin. The drug did not become widespread because it is only administered parenterally. It could only be used for short courses and also it causes a dose-dependent angiotensin II-similar effect. The negative experience of saralasin application slowed down searching of AT II receptors blockers for many years. At the same time studies of another class of drugs angiotensin-converting enzyme rapidly gaining turnover. The first drug of angiotensin II, approved for clinical use "losartan" was synthesized in 1995. It is a derivative of imidazole and not peptide derivative with high selectivity to the AT1 receptor. The class of drugs known as "sartans" (Zaharova et al. 2011, Shilov 2014).

Currently, the ARBs class (sartans) is represented by 8 drugs (losartan, valsartan, candesartan, telmisartan, eprosartan, irbesartan, olmesartan, azilsartan). Based on the results of large clinical trials, the presence of general sartans' class-effects can be considered. Chief among them is stable and sustained blood pressure control. In addition, across a range of studies have been received additional angiotensin-independent organ protective effects of sartans: cardioprotection (LIFE, JIKEI-HEART), nephroprotection (IRMA II, IDNT, MARVAL, RENAAL, DETAIL), neuroprotection (MOSES, ACCESS), improvement of glycemic control (VALUE, LIFE, ALPINE, NAVIGATOR) (Mancia et al. 2013, Kirichenko 2012).

The structure of most sartans is imidazole ring which provides the basic antihypertensive class-effect. Classeffects of drugs associated with blockade of physiologically significant influences of AT II, which are implemented through the AT1-receptors. The specific antagonistic action relative to AT1-receptors by the negative feedback mechanism increases the concentration of renin and AT II, aldosterone concentration decreases in plasma. ARBs II do not inhibit ACE and thus have no effect on the metabolism of bradykinin, do not cause cough. Sartans can be divided into two groups: prodrugs with active metabolites and drugs. Prodrugs include losartan, candesartan, olmesartan, and azilsartan (Chazova et al. 2011, Zaharova et al. 2011).

A very important property of sartans is the ability to reduce the degree of ventricular hypertrophy and cause regression of its remodeling, which are among the main predictors of early cardiovascular morbidity and mortality. The presence of electrocardiographic evidence of left ventricular hypertrophy increases risk of coronary heart disease 3-5 times, congestive heart failure - in 6-17 times regardless of blood pressure (Svischenko et al. 2012). ARBs increasingly used in the treatment of patients with paroxysmal atrial fibrillation (AF), except antiarrhythmic drugs in recent years. Reducing the risk of AF in the application of angiotensin II receptor blockers also ensured through their positive effects on sympathoadrenal system, heart rate variability, cardiac conduction system of the heart and systemic and intracardiac hemodynamics. With the ability of ARBs II to reduce the frequency of AF paroxysms can link the most pronounced reduction in the risk of stroke (up to $49 \%$ in patients with AF. It was also established in some studies that sartans slows the progression of coronary atherosclerosis, demonstrates advantage over ACE inhibitors on restenosis prevention and new lesions in coronary arteries in patients after stenting (Tseluyko et al. 2014). Equally important are nephroprotective properties of ARBs II. Clinical studies ROADMAP, MARVAL, PRIME, IDNT, DETAIL, PROTECTION and others had shown that treatment with ARBs II slows the progression of chronic kidney disease in patients with hypertension and type 2 diabetes, reducing the risk of microalbuminuria, distancing approach of terminal renal failure and increasing the duration of predialysis period (Shilov 2014, White et al. 2011).

One of the important aspects of drug-specific application of sartans is reduction in the incidence of type 2 diabetes. ARBs II are partial agonists of PPAR- $\gamma$ (peroxisome proliferatoractivated receptor- $\gamma$ ) - central regulator 
of insulin and glucose metabolism that increases the insulin sensitivity. Due to the stimulating effect increase the plasma level of adiponectin, decrease the severity of fatty liver steatosis, and dyslipidemia. Also described cerebroprotective properties, anti-inflammatory effects of sartans that are realized through reduction of proinflammatory cytokines tumor necrosis factor $\alpha$ and interleukin- 6 in plasma of patients suffering from type 2 diabetes combined with hypertension (Ambrosova et al. 2010, Sirenko 2012).

Pharmaceutical company "Takeda" that created the drug candesartan, has developed a modern angiotensin II receptor blocker possessing improved pharmacokinetics, drug azilsartan medoxomil under the trade name edarbi.

After oral administration azilsartan medoxomil is converted to the active form azilsartan, highly selective ARBs, by ether hydrolysis in the gastrointestinal tract and the liver. Bioavailability is $60 \%$, it reaches maximum concentration in the blood for 1.5-3 hours. The half-life accounts for 11 hours, which allows the drug to be taken 1 per day. More than $99 \%$ of the drug is bound to plasma proteins, mainly serum albumin. Azilsartan metabolized by cytochrome CYP2C9 via the formation of two inactive metabolites (MI and M-II). $55 \%$ of the drug is excreted from the body through the gastrointestinal tract, $42 \%$ - by kidneys (Savustyanenko 2014, Azilsartan medoxomil (Edarbi) the eighth ARB 2011).

Azilsartan is a competitive reversible antagonist of AT1 receptors. Azilsartan medoxomil showed minimal compared to other sartans medium inhibitory concentration (IC50), required for $50 \%$ inhibition of connection with receptor in experiments (Zaharova et al. 2011). This almost completely eliminates the possibility of angiotensin II binding to AT1 receptors, leading to pronounced inhibition of the renin-angiotensin-aldosterone system. As a result, azilsartan developing hypotensive effect faster, prolonged and pronounced compared to other sartans (Tseluyko et al. 2014, Kajiya et al. 2011). According to the clinical measurement of blood pressure and diurnal blood pressure monitoring showed statistically significant superiority in reducing BP compared to valsartan (White et al. 2011, Sica et al. 2011), candesartan (Rakugi et al. 2012) and olmesartan therapy (White et al. 2011, Bakris et al. 2011). The amount by which azilsartan medoxomil lowers blood pressure compared with the listed drugs may range from 1.2 to $\approx 5 \mathrm{~mm} \mathrm{Hg}$. This decline has some clinical significance: reduced systolic blood pressure by $2-5 \mathrm{~mm} \mathrm{Hg}$ could potentially reduce mortality from coronary heart disease in 4-9\%, stroke - by 6-14 \% (Mancia et al. 2013, Savustyanenko 2014, Baker et al. 2011).

Azilsartan acts quickly: within 2 weeks of therapy with edarbi leads to the same reduction in blood pressure as valsartan therapy for 4 weeks (Tseluyko et al. 2014, Sica et al. 2011, Baker et al. 2011).

In clinical use as a starting dose of azilsartan medoxomil $40 \mathrm{mg} 1$ per day is recommended. With the lack of efficacy, the dose may be increased to $80 \mathrm{mg} /$ day. In patients with mild/moderate renal impairment dose adjustment is not required. In elderly patients ( $\geq 75$ years) patients with mild/moderate hepatic impairment or low blood volume starting dose should be $20 \mathrm{mg} /$ day. In severe hepatic insufficiency drug use is not recommended, in severe renal insufficiency used with caution. The drug is not excreted in hemodialysis. To pregnant women drug is not administered due to its penetration through the placental barrier (Savustyanenko 2014, Kajiya et al. 2011).

The main trend in the treatment of sartans class preparation is the use of the drugs in high doses, the maximum tolerated permitted. For example, during recent clinical studies of azilsartan comparative effectiveness and safety in patients with hypertension were applied dose of azilsartan $80 \mathrm{mg}$, olmesartan $40 \mathrm{mg}$ and valsartan $320 \mathrm{mg}$ (Zaharova et al. 2011).

In addition to antihypertensive action azilsartan medoxomil shows a number of additional pleiotropic effects. These include antithrombotic, antiproliferative and antyfibrotic action. It demonstrates the improvement in glucose tolerance and tissue insulin sensitivity, increasing the expression of PPAR- $\gamma$ and adiponectin (Baker et al. 2011), improves endothelial function, reduces the progression of albuminuria, proteinuria (Shilov 2014, Savustyanenko 2014).

To enhance the antihypertensive action azilsartan medoxomil may be combined with other antihypertensive agents, such as chlorthalidone, hydrochlorothiazide, amlodipine, etc. (Dyadyk et al. 2014, Baker et al. 2011).

\section{Conclusions:}

1. The current strategy of high blood pressure correction provides effective blood pressure control with decreased risk of cardiovascular complications, lethal outcomes, improvement of the state of organs targets.

2. ARBs II - drugs with pleiotropic pharmacological properties that have a multicomponent antihypertensive efficacy, good tolerability, diverse organoprotection (improvement of cardiovascular, cerebrovascular and renal status), is highly safe, it can improve the remote prognosis for patients with hypertension. Sartans make up worthy competition with ACE inhibitors - a standard RAAS-acting drugs, but is dominated by recent criteria of safety, tolerance, ability to provide organoprotection. 
3. The high antihypertensive activity and a favorable safety profile of azilsartan medoxomil may provide better long-term compliance with therapy and optimal control of blood pressure in patients.

4. Promising is the use and further development of angiotensin II receptor blockers, as well as new classes of inhibitors of the RAAS: AT II-vaccines and vasopeptidase inhibitors.

\section{References:}

1. Ambrosova T., Kovalyova O., Ashcheulova T. (2010), The therapeutic potential of angiotensin II receptor antagonists in hypertensive patients with metabolic disorders: effect on carbohydrate and lipid profiles, Ukrainian Journal of Therapeutics. 4, 76-81.

2. Azilsartan medoxomil (Edarbi) the eighth ARB (2011), Med. Lett. Drugs Ther. 53 (1364), 39-40.

3. Baker W., White W. (2011), Azilsartan medoxomil: a new angiotensin II receptor antagonist for treatment of hypertension. The Annals of Pharmacotherapy, 45, 1506-1515.

4. Bakris G., Sica D., Weber M., White W.B., Roberts A., Perez A., Cao C., Kupfer S. (2011), The comparative effects of azilsartan medoxomil and olmesartan on ambulatory and clinic blood pressure. J. Clin. Hypertens., 13 (2), 81-88.

5. Chazova I., Martynyuk T., Ratova L. (2011), The focus of attention - angiotensin converting enzyme inhibitors: ramipril in hypertension. Systemic Hypertensions, 8 (3), 5-10.

6. Dyadyk A., Bahriy A., Chomenko M. et al. (2014), Hypertension in 2014: classification, diagnosis, treatment. News of Medicine and Pharmacy, 482, 53-69.

7. Kajiya T., Ho C., Wang J., Vilardi R., Kurtz T.W. (2011), Molecular and cellular effects of azilsartan: a new generation angiotensin II receptor blocker. J. Hypertens, 29 (12), 2476-2483.

8. Kirichenko A. (2012), Indications for use of AT1-receptor blockers in patients with hypertension. Handbook of Outpatient Physician, 10, 6-8.

9. Korost Y., Bula L., Suprunyuk V. (2014), The application features angiotensin II receptor blockers in the treatment of cardiovascular diseas. Medicines of Ukraine, 1 (177), 19-23.

10. Mancia G., Fagard R., Narkiewicz K. et al. (2013), 2013 ESH/ESC Guidelines for the management of arterial hypertension. European Heart Journal, 34, 2159-2219.

11. New therapeutic possibilities of control of hypertension: a modern angiotensin receptor antagonist - Edarbi (azilsartan medoxomil) (2014), Consilium Medicum, 10, 30-31.

12. Ostroumova O., Smolyarchuk E., Khvorostyanaya I. (2010), New approaches to the treatment of hypertension: the choice of the optimal drug to the choice of the optimal combination. Rational Pharmacotherapy in Cardiology, 6 (5), 709-716.

13. Rakugi H., Enya K., Sugiura K., Ikeda Y. (2012), Comparison of the efficacy and safety of azilsartan with that of candesartan cilexetil in Japanese patients with grade I-II essential hypertension: a randomized, double-blind clinical study. Hypertens. Res., 35, 552-558.

14. Savustyanenko A. (2014), New perspectives in achieving blood pressure control: focus on new sartan - azilsartan medoxomil. Hypertension, 4 (36), 23-27.

15. Shilov A. (2014), Sartans in the practice of primary health care in the treatment of hypertension. Farmateka, 9 (282), 39-44.

16. Sica D., White W., Weber M., Bakris G.L., Perez A., Cao C., Handley A., Kupfer S. (2011), Comparison of the novel angiotensin II receptor blocker azilsartan medoxomil vs valsartan by ambulatory blood pressure monitoring. J. Clin. Hypertens, 13(7), 467-472.

17. Sirenko Y. (2012), Angiotensin II receptor blockers in the treatment of hypertension: myths and reality. Hypertension, 4 (24), http://www.mif-ua.com/archive/article/33325 (accessed: 2013.03.05).

18. Svischenko E., Bezrodna L. (2012), Angiotensin II receptor blockers in the treatment of hypertension, Publisher O. Zaslavsky.

19. Tseluyko V., Ocheretyana N. (2014), The new representative of the sartans group azilsartan medoxomil (Edarbi): equal among equals or special? Health of Ukraine. Cardiology. Rheumatology. Heart Surgery, 4, 18-19.

20. White W., Weber M., Sica D., Bakris G.L., Perez A., Cao C., Kupfer S. (2011), Effects of the angiotensin receptor blocker azilsartan medoxomil versus olmesartan and valsartan on ambulatory and clinic blood pressure in patients with stages 1 and 2 hypertension. Hypertension, 57, 413-420.

21. Zaharova N., Kuz'mina-Krutetskaya S. (2011), Clinical pharmacology of sartans: class-effect and pharmacodynamic characteristics of drugs. Systemic Hypertensions, 8 (3), 10-16.

Submitted: 28.04 .2015

Accepted: 13.07.2015 